\title{
Linx
}

Revue des linguistes de l'université Paris X Nanterre

$7 \mid 1995$

Saussure aujourd'hui

\section{Saussure aux prises avec la notion de littérature}

\section{Michel Arrivé}

\section{(2) OpenEdition \\ Journals}

Édition électronique

URL : http://journals.openedition.org/linx/1136

DOI : 10.4000/linx.1136

ISSN : 2118-9692

\section{Éditeur}

Presses universitaires de Paris Nanterre

\section{Édition imprimée}

Date de publication : 1 septembre 1995

Pagination : 156-172

ISSN : 0246-8743

\section{Référence électronique}

Michel Arrivé, "Saussure aux prises avec la notion de littérature », Linx [En ligne], 7 | 1995, mis en ligne le 12 juillet 2012, consulté le 20 avril 2019. URL : http://journals.openedition.org/linx/1136 ; DOI :

$10.4000 / \operatorname{linx} .1136$

Ce document a été généré automatiquement le 20 avril 2019.

Département de Sciences du langage, Université Paris Ouest 


\title{
Saussure aux prises avec la notion de littérature
}

\author{
Michel Arrivé
}

1 Je souhaite commencer mon intervention par un aveu de perplexité. Cela fait de nombreuses années - près d'une vingtaine, sauf erreur - que je réfléchis autour des problèmes annoncés par le titre de ma communication. Je crois leur avoir consacré une petite dizaine d'articles. Je continue obstinément à relire Saussure, parfois à le lire, sans préfixe, quand une publication nouvelle, par exemple celle de Marinetti et Meli 1986 (désormais $L G M M$ ), rend enfin commodément accessibles des textes jusque là à peu près complètement inédits. Au fur et à mesure que je travaille, j'y vois de moins en moins clair. En sorte que ma communication ne sera rien d'autre que l'exposé, plus ou moins ordonné, plus ou moins hiérarchisé, de mes perplexités.

\section{Première perplexité}

2 Elle est déterminée par la difficulté que j'éprouve à articuler et, plus encore, à concilier, deux constatations que j'énonce d'emblée de façon sommaire, avant de préciser chacune d'elles :

3 La notion de littérature occupe dans le Cours de linguistique générale (désormais CLG) une place très marginale.

1. Les discours sur lesquels se porte de façon principale, voire presque exclusive, l'intérêt de Saussure dans ses autres recherches sont de type littéraire: les textes anagrammatiques et les textes légendaires.

4 En quoi l'énonciation de ces deux constatations peut-elle déterminer la perplexité ? Ne s'agit-il pas tout bonnement de la séparation légitimement opérée par Saussure entre deux champs de recherche séparés, celui de la langue et du langage pour le CLG, celui des textes, et spécifiquement des textes littéraires, pour ses autres travaux ? Cette solution ne tient pas. Pour la raison évidente que les textes littéraires relèvent du "discours" (Starobinski, 1971, désormais Ang, p. 14) et que celui-ci, présupposant « la langue », entre 
par là dans le champ d'investigation du linguiste, ou à tout le moins du sémiologue. On verra d'ailleurs plus bas que Saussure pose explicitement l'appartenance de certains textes à la sémiologie. La perplexité reste donc légitime. Il convient, pour en préciser la portée, d'examiner de façon un peu détaillée les deux propositions qui la déterminent :

1) La place de la littérature dans le CLG est-elle vraiment

À coup sûr, la notion de littérature n'est pas totalement absente du CLG. Le nom même de la littérature apparaît au moins trois fois (p. 41, 267 et 278). Mais la littérature n'est en rien alléguée pour elle-même. Il n'y a dans le CLG que deux exemples littéraires. Encore n'ontils que la fonction modeste d'illustrer deux faits lexicaux: l'innovation analogique de Rousseau, qui emploie traisait au lieu de trayait comme imparfait de traire (p. 231) et la formation, par Lactance, de meridionalis au lieu de meridialis (p. 233). Quant au bref passage sur la versification (p. 60), il a lui aussi une visée strictement linguistique. C'est dire à quel point la spécificité proprement littéraire des textes est occultée. Les allusions cursives à la littérature n'ont d'autre fonction que de marquer la part qu'elle a dans la constitution de la «langue littéraire ». Part, à vrai dire, non exclusive. Car on s'aperçoit que la « langue littéraire » ne se confond pas avec la « langue de la littérature »:

«La langue littéraire dépasse de toute part les limites que semble lui tracer la

littérature : qu'on pense à l'influence des salons, de la cour, des Académies » (p. 41).

La langue littéraire, pour Saussure, c'est - il le dit explicitement p. 267 - la «langue cultivée ». Et cette « culture » de la langue - au sens où l'on cultive une plante - s'opère essentiellement dans et par l'écriture. Au point qu'il semble, en certains points, qu'en viennent à se confondre les deux notions d'écriture et de littérature, comme si littérature était pris en son sens étymologique d'écriture, et littéraire au sens de littéral. Ainsi il est question p. 53 des «idiomes très littéraires, ou le document écrit joue un rôle considérable ». Conséquence inéluctable de cette relation entre langue littéraire et écriture : le statut problématique d'une langue littéraire sans écriture. Saussure signale rapidement le problème p. 269, à propos des poèmes homériques.

Ainsi, la littérature n'est alléguée très fugitivement dans le CLG que comme élément de la « langue littéraire ", elle-même liée à l'écriture, et par là à l'« artificiel », au «factice », à l'« externe » (voir p. $† 42$ et 46$)$, en opposition avec le caractère «naturel » de la « langue vulgaire ", la seule à relever du « système interne » (p. 192 et 267).

2) Les textes anagrammatiques et les textes légendaires sont-ils littéraires ? C'est un fait que le lecteur contemporain les considère comme tels. Mais en allait-il de même pour Saussure?

11 Parmi les textes qui lui offrent des anagrammes, Saussure fait des textes «littéraires » une sous-classe spécifique. Ils ont pour auteurs des « littérateurs proprement dits » (Ang, p. 26) , voire, pour certains d'entre eux, «les plus gens-de-lettres des littérateurs» (p. 116). Ils se distinguent des autres textes anagrammatiques, par exemple religieux ou funéraires, dont l'auteur n'est pas un «littérateur ", mais un vates, auteur de vaticinia. Cependant, la différence typologique entre les deux sous-classes de textes anagrammatiques n'est pas fixée avec rigueur. Saussure envisage explicitement des textes «en partie littéraires, comme ceux d'Andronicus et Nævius » (p. 21), sans s'expliquer sur les traits qui distinguent les parties ou les aspects littéraires et non littéraires.

Quoi qu'il en soit de leur spécificité, les textes anagrammatiques se caractérisent tous par leur «attachement à la lettre » (Ang, p. 38). On trouve ici un point commun entre 
l'enseignement du CLG et la recherche sur les Anagrammes: le lien indissoluble entre lettre et littérature. On ne peut s'empêcher de penser à la célèbre formule de Jarry : « Il n'y a que la lettre qui soit littérature ".

13 Qu'en est-il maintenant de la littérature - au sens saussurien - dans la recherche sur la légende? En de nombreux points de LGMM, les textes étudiés sont qualifiés d'« œuvres littéraires ». Là encore s'affiche de façon redondante la relation entre littérature et écriture . Ainsi dans le titre d'un projet de chapitre sur La légende de Dietrich (p. 250), l'adjectif littéraire est commenté par écrite. Et Saussure éprouve encore le besoin de préciser davantage, par un sous-titre redondant : «Division des monuments écrits comme œuvres écrites" (ibid.). Ici apparaît une difficulté: Saussure laisse entendre plus ou moins clairement que la légende n'est littéraire que lors de son accès à l'écrit. Tant qu'elle n'est envisagée que dans sa manifestation orale, elle n'accède pas - en dépit, çà et là, de certaines hésitations terminologiques, par exemple p. 198 - au statut littéraire. Le syntagme «littérature orale » reste pour lui, si j'ai bien lu, un oxymore impossible. La distinction est, pour l'essentiel, posée entre la légende dans sa manifestation orale donnée comme étant par nature évolutive - et les «élaborations littéraires ", voire les " altérations littéraires » (p. 283) dont elle a été l'objet lors de sa fixation par écrit. Entre ces deux objets semble s'établir une hiérarchie analogue à celle qui s'observe dans le CLG entre « la langue naturelle », orale, et « la langue littéraire », fixée et " réglementée » par l'écriture. Le cas particulier du Nibelungenlied, du moins dans la réflexion saussurienne ${ }^{1}$, est qu'il fixe la légende dans son état originel, en sorte que pour ce texte, et pour ce texte seulement parmi ceux que Saussure envisage, se neutralise l'opposition entre légende et littérature: il est à la fois légendaire et littéraire. C'est du moins de cette façon que j'interprète le passage, inachevé et énigmatique, de la page 441 :

14 «Ce qui fait <la valeur, incomparable> immensité du Nibelungenlied, ce n'est pas comme pour Homère l'antériorité de date sur une littérature, car le texte <peut passer pour être> de 1190 et à peine antérieur à des productions comme le Biterolf, mais c'est donner la légende en sa forme originale et <ENCORE> PRISE <comme une ? qui se dit> AU SÉRIEUX ».

En somme, ma première perpexité subsiste pleinement. J'ajoute même qu'elle s'épaissit un peu plus quand je compare l'énumération des objets possibles de la « sémiologie » dans le $C L G$ et dans les $L G M M$.

Dans le CLG, p. 33, ces objets sont "l'écriture, l'alphabet des sourds-muets, les rites symboliques, les formes de politesse, les signaux militaires». Il est vrai qu'un "etc» redoublé marque que cette liste d'objets n'est pas close. Mais l'édition critique de Rudolf Engler (p. 46-47) comme les Sources Manuscrites de Godel (p. 66) et le Cahier de Constantin publié par Komatsu (p. 324-325) donnent des listes identiques, à des détails de formulation près, et n'explicitent pas le «etc» de la version standard. Jamais la littérature, ni même aucun objet discursif, littéraire ou non, n'apparaît dans cette énumération.

Inversement, la légende est explicitement donnée, dans les LGMM, comme objet de la « sémiologie ", exactement au même titre que « les mots de la langue »:

- La légende se compose d'une série de symboles<dans un sens à préciser.>

- Ces symboles, sans qu'ils s'en doutent, sont soumis aux mêmes vicissitudes et aux mêmes lois que toutes les autres séries de symboles, par exemple les symboles qui sont les mots de la langue.

- ils font tous partie de la sémiologie » (p. 30 ; le tous semble bien embrasser les «symboles " de la légende et ces autres « symboles » qui sont les mots de la langue). 
L'aveu de ma première perplexité n'aura pas été absolument inutile. Il nous a en effet permis de repérer deux points communs entre les trois ensembles de textes saussuriens :

1. La constance et la force de la relation établie, de la même façon dans les trois recherches, entre les notions de littérature et d'écriture. Parlons désormais, en termes non saussuriens, de la littéraLité de la littérature.

2. La reconnaissance, manifestée également dans les trois textes, quoique, cette fois, sous des formes différentes, d'une spécificité de la littérature. Il existe pour Saussure une classe spécifique de discours, typologiquement distincts des autres discours, même si la différenciation typologique n'est pas explicitée avec précision. Ces discours spécifiques, ce sont, dans sa terminologie, les "œuvres littéraires ». Pour désigner leur spécificité, je m'autoriserai là aussi l'emploi d'un terme non saussurien et, qui plus est, anachronique : la littéRarité.

Qu'en est-il, chez Saussure, de la littéralité et de la littérarité de la littérature ? Ces deux questions vont faire l'objet de la suite de mon exposé. Elles prendront, c'est à craindre, la forme de nouveaux aveux de perplexités. Il est possible également qu'elles en viennent à se rencontrer : il n'y a en somme entre les deux notions que la différence d'une... lettre.

\section{Deuxième perplexité}

Elle est relative à la littéraLité, chez Saussure, de la littérature. Quelle est précisément la forme de la relation entre littérature et écriture ? Il est ici indispensable de faire un bref détour par le problème du statut de l'écriture dans la réflexion saussurienne. On sait que s'observent dans le CLG deux attitudes différentes, voire, d'une certaines façon, opposées, à l'égard de l'écriture :

21 En certains points du CLG, l'écriture est donnée comme fondamentalement seconde par rapport à la manifestation orale. C'est le célèbre passage des pages 44 et 45 , qui pose que « l'objet linguistique n'est pas défini par la combinaison du mot écrit et du mot parlé : ce dernier à lui seul constitue cet objet ", en sorte que "l'écriture [est] en elle-même étrangère au système interne ». De cette mise en place des relations entre écrit et oral découlent les célèbres jugements dépréciatifs portés, p.51-53, sur l'écriture, et notamment l'analyse du cas "tératologique» de Levèfre - Lefebvre - Lefébure (p. 53-54).

Présupposé évident de cette analyse des relations entre écrit et oral : la conceptualisation du signifiant comme exclusivement sonore. Qu'il suffise ici de rappeler que, dans la mise en place du concept de signe, le terme signifiant ne fait que se substituer à la notion, préalablement posée, d'image acoustique.

23 Mais on sait que ce point de vue sur le statut de l'oral et, par voie de conséquence, sur ses relations avec l'écrit est fortement modifié en d'autres points du CLG. Loin d'être constitué par la substance vocale et sa manifestation acoustique, "le signifiant linguistique n'est aucunement phonique: il est incorporel»(p. 164). Selon les Sources manuscrites du Cours II, Saussure posait explicitement le problème de «l'indifférence du moyen de production »:

« <C'est moins évident>. Est-il si nécessaire que la langue se prononce par l'organe vocal ? Non : les mots peuvent être transposés dans l'écriture. L'instrument n'y fait rien. Ainsi la comparaison de la langue avec un autre système de signes nous permet d'aller jusque là, d'affirmer que là n'est pas l'essence de la langue »(Godel, 1969, p. 193-194 ; Engler, 1989, p. 270).

24 Ainsi se dissout la notion de «secondarité ", d'« extériorité » de l'écriture. L'écriture accède pleinement au statut de « système de signes ", et justifie par là son appartenance 
aux objets sémiologiques. D'où l'utilisation de l'exemple de la lettre - spécifiquement le T est ses diverses réalisations - pour illustrer la notion de valeur².

Je serai muet sur les difficulté posées par cette duplicité de la réflexion saussurienne. Je m'installerai dans la seconde conception de l'écriture, celle qui fait d'elle un système de signes "similaire à celui de la langue ». La question que je me poserai est apparemment vétilleuse, mais en réalité fondamentale : qu'en est-il du signifiant graphique à l'égard du "second principe » qui gouverne le signe, le «caractère linéaire du signifiant " (CLG, p.103) ? Cette question sera successivement posée à propos de trois types de discours : celui de la "langue naturelle», celui des textes anagrammatiques et celui des textes légendaires.

Avant d'entreprendre ce triple examen, il convient toutefois d'au moins signaler une équivoque, qui se manifeste par une question : quel est au juste, dans le CLG, l'objet affecté par le «caractère linéaire»? Est-ce le signifiant seul - comme semble l'indiquer le décalage opéré entre les deux " principes » : « arbitraire du signe » - « caractère linéaire du signifiant»? Est-ce l'enchaînement des signes dans le discours, comme semble l'indiquer le raisonnement mis en place p. 170 pour fonder la notion de syntagme ? En ce point, Saussure fait apparaître le " caractère linéaire de la langue », évidemment distinct $\mathrm{du}$ « caractère linéaire du signifiant ».

Plutôt que d'envisager de front ce difficile problème ${ }^{3}$, il vaut mieux s'installer confortablement dans l'ambiguïté saussurienne, et en venir à l'examen de la question annoncée, pour chacun des trois types de discours.

28 1) Les discours écrits de la " langue naturelle ». Ici, aucune hésitation : ils sont pleinement soumis à la linéarité. Saussure le signale explicitement dès la page 103, quand il remarque que «le caractère [linéaire] apparait immédiatement dès qu'on représente [les signifiants] acoustiques par l'écriture et qu'on substitue la ligne spatiale des signes graphiques à la succession dans le temps ». Des exemples sont fournis p. 190 :

«Le sens du français désir-eux ou du latin signi-fer dépend de la place respective des sous-unités : on ne saurait dire eux-désir ou fer-signum »

On aura remarqué qu'entre les deux fragments cités on est passé de la linéarité du signifiant («les signes graphiques») à la linéarité des signes dans l'enchaînement du syntagmes : car ce sont bien des signes que désir et -eux, que signum et -fer. Mais ce n'est pas cette contradiction - si c'en est une ${ }^{4}-$ qui, pour l'instant nous intéresse. Je me contente de conclure, pour une fois de façon assurée: oui, les discours écrits de la «langue naturelle» sont soumis à la linéarité, sans que soit envisagée la moindre exception à cette soumission.

30 2) Pour les textes anagrammatiques, il va être nécessaire d'entrer dans le détail de la manipulation saussurienne du signifiant. Je vais prendre un exemple bien connu des lecteurs de Ang (p. 65) : c'est le vers saturnien

DONOM AMPLOM VICTOR AD MEA TEMPLA PORTATO, extrait du vaticinium "Aquam albanam » cité par Tite-Live et reconstitué par Saussure sous l'aspect « archaïque » qu'il devait avoir lors de sa formulation, en 397.

Pour le texte de surface, celui qui constitue le vers manifeste, aucun problème : il est, comme tout segment d'une langue naturelle, soumis à la linéarité. Qu'en est-il du texte anagrammatisé ? Comme souvent ${ }^{5}$, il se réduit ici à un nom propre, celui du dieu APOLO, pour lequel Saussure commence par admettre l'orthographe avec un seul L. Il examine 
d'abord le second hémistiche. Le nom recherché s'y trouve disséminé de la façon suivante :

\section{AD MEA TEMPLA PORTATO A $\quad$ PL $\quad \mathrm{O} \quad \mathrm{O}$}

On le voit: les lettres d'APOLO apparaissent «dans le désordre »: il faut transférer le premier $\mathrm{O}$ devant le L qui le précède. À propos de cet hémistiche, Saussure se contente de remarquer, sans trop sembler s'en inquiéter, que le L est en contact avec APO, mais « du mauvais côté ».

Il est un peu plus explicite à propos du premier hémistiche, étudié en second lieu parce qu'il est, selon lui, « d'une qualité assez inférieure » (p. 71) :

\section{DONOM AMPLOM VICTOR A PLO

Ici, Saussure se sent contraint de signaler explicitement l'entorse à la linéarité : « On voit bien A initial et ensuite PLO, qu'on peut accepter pour POL » (p. 71).

J'insiste sur cette formulation: tout se passe comme si PLO valait pour POL, comme si, dans une autre langue, TUG était équivalent de GUT. Je ne donne pas cet exemple au hasard : c'est l'un de ceux que cite ... Freud dans sa célèbre spéculation, inspirée de Carl Abel, sur les «sens opposés des mots primitifs" (Freud, 1910[1971], p. 66) , où la métathèse sans incidence sémantique - c'est-à-dire la rupture de la linéarité - tient une place aussi importante que l'antithèse (voir Freud, 1910, p. 67 et M. Arrivé 1986 b).

De tels exemples sont-ils isolés dans la recherche sur les anagrammes? Point du tout : ils pullulent, pratiquement à toutes les pages, et Saussure manque rarement de les signaler, mais le plus souvent sans inquiétude explicite : «ROD est rendu par ORD » (p. 81), «RO par OR », « sans rigueur » (p. 85) ; « FLORES est pris comme FROLES », p. 82 ; «PR-T ou TPR pour TR-P est une transposition d'un caractère bénin » (p. 87). Parfois cependant, il semble perplexe, peut-être même vaguement désapprobateur. Il en vient par exemple à utiliser l'expression « tour de passe-passe » (p. 83), formule que je suis tenté de prendre à la lettre : les lettres passent les unes devant les autres, en dépit de toute linéarité.

Il y a plus spectaculaire encore : les deux éléments d'un mot composé peuvent se trouver « renversés dans leur ordre respectif » ( $p$ 52). Ainsi le nom propre composé HERACLITUS - où, pour l'helléniste et même le latiniste cultivé, les deux éléments HERA et CLITUS sont clairement identifiés comme signes distincts - est donné dans l'ordre CLITUS-HERA. En somme, Saussure donne là un exemple comparable à ceux de DÉSIREUX et de SIGNIFER. La différence est que dans le texte anagrammatique il devient possible scandaleusement - de disposer les deux unités dans un ordre indifférent : HERA-CLITUS et CLITUS-HERA, c'est la même chose.

En ce point Saussure est amené, d'une façon que je persiste à considérer comme exceptionnelle, à confronter les données qu'il met en œuvre dans la recherche 
anagrammatique et celles que lui livrent les principes de la linguistique. C'est le célèbre passage de la page 46 :

«Que les éléments qui forment un mot se suivent, c'est là une vérité qu'il vaudrait ne pas considérer, en linguistique ${ }^{6}$, comme une chose sans intérêt parce qu'évidente, mais qui donne au contraire le principe central de toute réflexion utile sur les mots ${ }^{7}$. Dans un domaine infiniment spécial comme celui que nous avons à traiter, c'est toujours en vertu de la loi fondamentale du mot humain en général que peut se poser une question comme celle de la consécutivité ou nonconsécutivité et dès la première ${ }^{8}$

Peut-on donner TAE par ta + te, c'est-à-dire inviter le lecteur non plus à une juxtaposition dans la consécutivité, mais à une moyenne des impressions acoustiques hors du temps? hors de l'ordre dans le temps qu'ont les éléments? hors de l'ordre linéaire qui est observé si je donne TAE par TA-AE ou TA-E, mais ne l'est pas si je le donne par ta + te à amalgamer hors du temps comme je pourrais faire pour deux couleurs simultanées? » (p. 46-47).

Depuis plus de vingt ans que, grâce à Starobinski, j'ai découvert ce texte, je le relis toujours avec admiration et presque émotion, frappé à la fois par son inachèvement comme si la pensée hésitait devant ses propres extrémités - et par la perfection formelle de certains fragments, par exemple cet alexandrin de type mallarméen, selon la formule du regretté Thomas Aron (1970) : «Hors de l'ordre dans le temps qu'ont les éléments ». Quoi qu'il en soit, il m'apparaît à l'évidence qu'ici le texte de la recherche fait écho avec précision au CLG . Le " caractère linéaire du signifiant » se trouve en effet précisément évoqué, et en même temps inversé. En linguistique, point d'exception au principe de la " consécutivité ", nom donné dans la recherche - de façon d'ailleurs plus pertinente - à ce qui est appelé "linéarité » dans le CLG. En revanche, dans «le domaine infiniment spécial» des Anagrammes, la question se pose: la consécutivité spatio-temporelle ne semble pas y être constante. D'où cette comparaison, in fine, avec les objets d'une sémiologie tabulaire : les lettres du texte anagrammatique seraient à «amalgamer hors du temps » à la manière des couleurs simultanées (et non successives) d'un tableau. On sait que ce type d'objet sémiologique est également évoqué dans le CLG (p. 103), mais précisément pour être opposé au fonctionnement du signifiant linguistique.

Nous venons d'apercevoir l'aspect littéral de la spécificité du texte anagrammatique : la lettre et, nécessairement, le phonème n'y sont pas soumis à la contrainte de la consécutivité. Ce régime spécifique de la littéralité anagrammatique constitue-t-il l'éventuelle littérarité de de ce type de texte ? Pour anticiper, j'indique qu'il en est à coup sûr l'un des éléments. Mais pas le seul : il faudra, un peu plus tard, poser la question des autres aspects spécifiques - non exclusivement littéraux - du texte anagrammatique. Je le ferai au moment de livrer ma troisième perplexité.

Avant d'y venir, il convient de poser la question de la linéarité - ou, mais on vient de voir que c'est tout un, de la consécutivité - dans le texte légendaire. En somme, le problème se ramène à cet autre : le texte légendaire donne-t-il dans la pratique de l' anagramme ? Là encore, la situation est particulièrement embrouillée, à la fois du point de vue philologique (pour la lettre des textes et pour leur édition) et du point de vue théorique. Pour y voir un peu clair, je distinguerai deux moments dans mon analyse. Je l'indique à l'avance : ils risquent de paraître donner lieu à des conclusions contradictoires.

43 1) À chaque instant dans le texte de la légende, on rencontre des manipulations sur la forme des noms propres qui évoquent plus ou moins le fonctionnement anagrammatique. Par exemple, p. 240, Saussure s'interroge sur des noms de villes cités dans le texte légendaire : 
«Freisach peut être Friesach en Carinthie. [...] Toutefois, rappelle d'une part Breisach, siège des Harlung et d'autre part Fridsaela (Verceil) par position géographique et par nom ».

l'aura compris à la prudence de mes formules : ce serait sans doute aller trop vite en besogne que de repérer un fonctionnement nettement anagrammatique dans ces analyses littérales des noms propres de la légende. Il faudrait naturellement, pour porter un jugement pleinement autorisé, s'infliger la tâche - hallucinante - d'étudier en détail tous les jeux de ce type dans les 450 pages des LGMM, sans parler de celles qui restent inédites. Les quelques sondages que j'ai faits me portent à hasarder trois remarques :

- les relations littérales de ce premier type dans la légende semblent ne se révéler qu'entre des noms propres, à la différence de celles qui s'observent dans les textes effectivement anagrammatiques, où elles s'établissent entre deux discours, dont l'un seulement - celui qui est anagrammatisé - peut se réduire à un nom propre. De ce fait, les relations littérales sont, en dépit de leur nombre, infiniment moins fréquentes dans la légende que dans la littérature anagrammatique, où elles sont par définition constantes ;

- les relations littérales de la légende s'observent fréquemment entre deux noms également présents dans le texte de surface. C'est par exemple le cas pour Sigmund et Sigismund, noms qui, en dépit de leur "identité ", désignent deux personnages différents, et apparaissent de ce fait alternativement à la surface du texte. Là encore ce caractère s'oppose au fonctionnement anagrammatique proprement dit, qui est "cryptographique ", c'est-à-dire « se rapporte à des noms ou à des mots qui ne sont pas prononcés au cours de la pièce » (Ang, p. 69).

- même dans les cas où les manipulations littérales repérées - ou construites - par Saussure semblent évoquer la pratique anagrammatique, les phénomènes de mise en cause de la linéarité du signifiant sont exceptionnels et même, semble-t-il, accidentels. Le seul exemple net est celui d' Adaocaro/Adoacaro/ Odoacre, pour lequel il faut vraisemblablement faire la part d'altérations textuelles liées aux aleas de la transmission des textes.

2) À côté de ces jeux apparentés de loin à la pratique anagrammatique, il existe au moins dans la description de Saussure quelques exemples d'anagrammes incontestables. Ils ont été publiés par Shepeard (1986) et commentés par Kim (1991, p. 274). Ansi le nom de Hagene se trouve anagrammatisé, exactement sur le même mode que celui d'Apolo dans le vaticinium Aqua albanam, par le vers 


\begin{tabular}{|l|l|l|l|l|l|l|}
\hline HOUBERT & ZE & GIBE & TRUËGE & {$[\ldots .]$.} & DEN & EZELN \\
\hline H[A] & G & E & GE & EN & E $^{9}$ & \\
\hline
\end{tabular}

Il n'y a en principe pas lieu de s' étonner de cette présence de l'anagramme dans la poésie germanique. C'est, d'une part, une constante selon Saussure, de la poésie indoeuropéenne archaïque. Et d'autre part la célèbre spéculation sur la "poésie germanique allitérante » et le nom allemand de la lettre (Buchstabe, Ang, p 38-40) rend compte de la possibilité de telles manipulations littérales.

J'observe toutefois que ce fonctionnement anagrammatique n'est pas retenu par Saussure comme fondamental dans la recherche sur la légende : il ne lui consacre que quelques pages, d'ailleurs bizarrement occultées, sans explication, dans les LGMM, p. $300^{10}$. Envahissante au point d'en devenir obsessionnelle dans les travaux sur la littérature gréco-latine, la quête des anagrammes est discrète et marginale dans la recherche sur la légende.

51 Ainsi, Saussure, d'un même mouvement, remarque la présence de la pratique anagrammatique dans le Nibelungenlied, et ne lui consacre qu'une part infime de sa réflexion. Quelle est la voie de la prudence devant ce qui reste, à mes yeux, une grave difficulté ? Elle consiste sans doute à poser que le discours légendaire n'est pas caractérisé comme spécifique par la pratique de l'anagramme. De ce fait, le fonctionnement qui lui est affecté par Saussure ne met pas fondamentalement en cause le principe de la linéarité du signifiant : même s'il comporte des anagrammes, il n'est pas lu comme anagrammatique. Il n'est retenu que dans son fonctionnement de surface, tout proche du discours de la langue naturelle. L'ensemble que constituent ces deux types de discours s'oppose à la littérature anagrammatique, qui bénéficie d'un régime littéral absolument «spécial» - pour reprendre l'adjectif saussurien : la mise en cause de la consécutivité.

52 Est-il possible de spéculer sur les raisons qui poussent Saussure à tenir cette position apparemment étonnante? Je courrai le risque de hasarder une hypothèse. Mais il faudra attendre que $\mathrm{d}$ ' autres aspects se soient révélés

53 J'en ai terminé avec l'aveu de ma deuxième perplexité. Il faut bien avouer que la conclusion à laquelle elle nous mène est doublement déconcertante. Car elle semble d'abord avoir pour effet de soumettre au même régime littéral des textes « littéraires »ceux de la légende, qui, en dépit de quelques précautions terminologiques, sont bien donnés comme tels - et des textes non littéraires: ceux du discours de la langue naturelle. Les uns et les autres se distinguent des textes anagrammatiques, généralement qualifiés comme « littéraires ». Mais elle doit, dans un second moment, être modulée par la présence réelle - quoique le plus souvent occultée dans la description qui en est faite - de structures anagrammatiques dans le texte légendaire.

On l'aura compris sans peine: ma troisième perplexité ne s'est pas dissipée lors de l'examen de la deuxième. Elle s'est même épaissie. Il subsiste beaucoup de pénombre du côté de la littéraLité. Du côté de la littéraRité, l'obscurité reste totale. C'est donc cette troisième - et ultime question - que je vais essayer de poser. 


\section{Troisième perplexité}

\section{Les problèmes que}

1. Le régime spécifique de la littéralité qui caractérise les textes anagrammatiques suffit-il à caractériser la littérarité de ces textes?

2. La disjonction opérée par Saussure ${ }^{11}$ du point de vue du fonctionnement littéral entre textes anagrammatiques et textes légendaires s'observe-t-elle également dans d'autres aspects de leur fonctionnement?

\section{Premier problème}

Le régime littéral des textes anagrammatiques est spécifique: leur signifiant n'est pas soumis de façon constante à la linéarité. Cette spécificité a-t-elle des implications au niveau du signifié ? À l'évidence oui : si le texte se dédouble au niveau du signifiant, il se dédouble nécessairement au niveau du signifié. Saussure insiste à différentes reprises sur cette question. Il va même jusqu'à distinguer le point de vue du "poète » de celui du « lecteur » : l'un et l'autre sont conscients du dédoublement du sens du texte. Le poète a pris soin, "avant tout, [de] se pénétrer des syllabes et combinaisons phoniques de toute espèce, qui se trouvaient constituer son thème» (p. 23). En somme, il travaille à disséminer à la surface du texte les éléments du thème qui est en réalité un autre texte, même si, souvent, il se réduit à un nom propre. Au point que ce «jeu a pu devenir l'accompagnement habituel, pour tout Latin qui prenait la plume, de la forme qu'il donnait à sa pensée presque à l'instant où elle jaillissait de son cerveau, et où il songeait à la mettre en prose ou en vers » (Ang, p. 120-121). Quant au lecteur, il est allégué - dans ce que j'ai lu - de façon plus discrète, précisément sous l'espèce du poète lui-même lisant d'autres poètes. Ainsi Saussure spécule-t-il sur la façon dont Virgile devait lire Homère :

« Un poète comme Virgile devait voir facilement des anagrammes répandus dans le texte d'Homère, il ne pouvait pas par exemple douter que dans un morceau sur Agamemnon un vers comme Aasen argaleon anemon amegartos autmè fût relatif par ses syllabes à Agamemnon » (p. 127).

Il y a plus encore. En un autre point, Saussure fait allusion à la «sociation psychologique inévitable et profonde» (p. 120) de la pratique anagrammatique. Pour qu'il y ait "sociation psychologique ", il faut bien qu'il y ait une convention établie dans la " masse parlante» - c'est intentionnellement que j'introduis ici le vocabulaire du CLG - pour faire reconnaître par tous la fondamentale polyphonie du texte littéraire.

On le voit: du point de vue de la littérarité, la spécificité anagrammatique réside indissolublement dans le régime de son signifiant et dans la polyphonie que ce régime implique.

J'indique au passage, plus timidement, une autre direction de recherche. Elle n'est indiquée par Saussure que de façon assez fugitive. On vient de voir que la pratique, active ou passive, de l'anagramme suppose la «sociation». Comme celle d'un système sémiologique ; comme celle, par exemple d'une langue. Essayons de pousser plus loin la comparaison. L'un des traits spécifiques de la langue dans le CLG est son caractère évolutif : point de langue qui ne soit à tout instant soumise au changement diachronique. Qu'en est-il de la pratique de l'anagramme? Saussure le remarque, avec ce que je crois 
pouvoir désigner comme une ombre de surprise : il n'évolue pas, il reste identique à luimême, sans la moindre mutation, pendant plusieurs siècles, voire millénaires :

«Depuis les plus anciens monuments saturniens jusqu'à la poésie latine qu'on faisait en 1815 ou 1820, il n'y a jamais eu d'autre manière d'écrire des vers latins que paraphraser chaque nom propre sous les formes réglées de l'hypogramme» $(\mathrm{p}$. 133 ; voir aussi, p. 119, une allusion à la transmission inchangée de la pratique « à travers les siècles et les milieux les plus différents de la culture latine »).

Ainsi l'anagramme se trouve-t-il faire exception non seulement à la linéarité, mais encore à l'évolution diachronique, c'est-à-dire aux deux modes saussuriens d'intervention du temps sur les objets du langage.

\section{Second problème}

Il ne nous reste qu'à nous interroger sur les raisons qui expliquent la disjonction opérée par Saussure dans le traitement qu'il confère aux textes anagrammatique et aux textes légendaires. Une réponse, pour une fois, semble s'imposer. Elle a déjà été aperçue. C'est que le texte légendaire, à la différence du texte anagrammatique, n'est en somme qu'accidentellement littéraire. Il ne fait que fixer, tardivement, et, par là, arrêter, à tous les sens du mot, un texte légendaire antérieur, qui présente, lui, tous les aspects sémiologiques de la langue - et fondamentalement la propension inéluctable au changement diachronique. Il se distingue par là du texte essentiellement littéraire qui, fixé d'emblée dans sa forme définitive, est par là empêché de connaître «l'épreuve du temps ni l'épreuve de la socialisation » (LGMM, p. 193), ces deux épreuves étant, pour Saussure, inséparables (voir le CLG, p.113). Ainsi s'explique l'apparent paradoxe de l'exclusion du texte "littéraire» du champ de la sémiologie: si le Don Quichotte de Cervantès - nommément cité p. 193 - n'est pas un objet sémiologique, c'est qu'il est éternellement fixé, sans possibilité aucune ni de socialisation, ni d'évolution diachronique. Il ne saurait pour cette raison être comparé à la langue. Il en va autrement pour le texte légendaire, qui s'égale en «noblesse » à la langue. D'où cette comparaison qui me parait fournir la meilleure des conclusions à cet ultime point de mon exposé :

«Ce qui fait la noblesse de la légende comme de la langue, c'est que condamnées l'une et l'autre à ne se servir que d'éléments apportés devant elles et d'un sens quelconque, elles les réunissent et en tirent continuellement un sens nouveau. Une loi grave préside, qu'on ferait bien de méditer avant de conclure à la fausseté de cette conception de la légende : nous ne voyons nulle part fleurir une chose qui ne soit la combinaison d'éléments inertes, et nous ne voyons nulle part que la matière soit autre chose que l'aliment <continuel> que la pensée digère, ordonne, commande, mais sans pouvoir s'en passer » (LGMM, p. 307).

C'est en ce point ultime qu'on peut se hasarder à spéculer. C'est, peut-être, cette fondamentale évolutivité de la légende qui rend compte du sort fait par Saussure à ce qui peut s'observer de pratique anagrammatique dans le texte littéraire qui en est issu. Car l'anagramme, comme on vient de l'apercevoir, échappe à l'évolution diachronique: comment pourrait-il s'instituer et, surtout, subsister dans un discours en perpétuel mouvement, qui, d'« un sens quelconque[...] tire continuellement un sens nouveau » ? Il y aurait en somme incompatibilité théorique entre légende et anagramme. Il est vrai que la légende finit par s'écrire et par donner lieu, mais en fin de parcours, à un texte littéraire, qui la fixe. Dès lors l'anagramme peut apparaître. Mais il n'affecte que l'« élaboration littéraire ", et non la légende qui en est le support: c'est pourquoi Saussure en signale 
discrètement la présence. Il se garde toutefois d'insister sur un trait qui ne caractérise pas l'objet qu'il s'est donné, mais la forme ultime que l'écriture lui a donnée.

\section{Note récapitulative}

63 J'ai laissé Saussure conclure lui-même. Il me reste, plus modestement, à essayer de récapituler les points repérés au cours de l'énumération de mes perplexités. Certes, de nombreuses questions restent pendantes : c'est sans doute pour une part l'effet de l'état d'inachèvement des textes saussuriens. Il faut parfois en venir à faire parler le silence de Saussure... Je me le suis permis une ou deux fois, sans doute imprudemment. Cependant certaines lignes de force se dégagent avec une apparence de certitude :

- le lien indissoluble du littéraire et du littéral ;

- la nature fondamentalement évolutive des authentiques objets sémiologiques, tels que ces deux très proches parents : le signe de la langue et le symbole de la légende;

- inversement, l'insensibilité au temps du texte "littéraire». D'une façon générale, il n'est pas soumis à la diachronie. Sous sa forme anagrammatique, il échappe non seulement à l'évolution dans le temps, mais aussi à la soumission à la consécutivité. Il se construit par là comme objet monstrueux, qui met en cause les principes mêmes de la sémiologie, et de ce fait lui échappe.

\section{BIBLIOGRAPHIE}

Aron, Thomas, 1970, « Une seconde révolution saussurienne? ", Langue française, 7, septembre, p 56-62.

Arrivé, Michel, 1986 a, « Intertexte et intertextualité chez Ferdinand de Saussure ", in Theis, R. et Sieppe, H.-T., Le Plaisir de l'Intertexte, Berne, Peter Lang, p. 11-31.

Arrivé, M., 1986 b, Linguistique et psychanalyse : Freud, Saussure, Hjelmslev, Lacan et les autres, Paris, Méridiens-Klincksieck.

Engler, Rudolf, voir Saussure 1989.

Freud, Sigmund, 1910 [traduction française 1971], « Des sens opposés dans les mots primitifs », Essais de psychanalyse appliquée, Paris, Gallimard, p. 59-67.

Godel, Robert, 1969, Les sources manuscrites du Cours de linguistique générale de Ferdinand de Saussure, Genève, Droz.

Kim, Sungdo, 1991, Ferdinand de Saussure : de la langue au mythe, thèse de l'Université de Paris-XNanterre.

Komatsu, Eisuke, voir Saussure 1987.

Marinetti, Anna et Melo, Marcello, voir Saussure 1986.

Milner, Jean-Claude, 1989, Introduction à une science du langage, Paris, Le Seuil.

Saussure, Ferdinand de, 1916 [1922,1986], Cours de linguistique générale, Paris, Payot. Abréviation : CLG. 
Saussure, F. de, 1986, Le leggende germaniche, Scritti scelti e annotati a cura di Anna Marinetti e Marcello Meli, Este, Libreria editrice di Zielo. Abréviation : LGMM.

Saussure, F. de, 1987, Cours de linguistique générale (III) (Cahier de Constantin), édité par Komatsu, Eisuke, Annual collection of Essays and Studies, Tokyo, Gakushuin University, vol. XXXIV, p. 305-411.

Saussure, F. de, 1989, Cours de linguistique générale, édition critique par Rudolf Engler, t. I, Wiesbaden, Otto Harrassowitz.

Shepeard, David, 1986, « Saussures Anagramme und die deutsche Dichtung », Schprachwissenschaft, 11, p. 52-67.

Starobinski, Jean, 1971, Les mots sous les mots. Les anagrammes de Ferdinand de Saussure, Paris, Gallimard. Abréviation : Ang.

\section{NOTES}

1. Est-il nécessaire de préciser qu'il n'est pas question ici de s'interroger sur l'« exactitude » de cette analyse?

2. Une résurgence inattendue de la première conception semble apparaître, p. 165, dans l'expression « le son que la lettre désigne ». Mais les sources manuscrites révèlent que Saussure a effectivement dit « la chose à désigner » ou « la chose qu'il [le signe graphique] veut désigner » (Godel, 1969, p. 193 ; Engler, 1989, p. 269). Ce qui change tout : car cette « chose », ce n'est pas le son, mais le «signifiant incorporel»: il se trouve alternativement manifesté par le son et par la lettre, qui accèdent de ce fait à un statut identique.

3. Milner $(1989$, p. $385,389,391)$ va jusqu'à parler comme d'une notion saussurienne de «la linéarité du langage », ce qui fait au plus haut point problème (par rapport à Saussure, certes, mais aussi de façon générale).

4. On peut en tout cas en expliquer l'origine : tout part de la linéarité des unités du signifiant, phonèmes ou lettres. Cette linéarité se réalise de la même façon au sein d'un signe (par exemple dans désir) et aux frontières de deux signes (par exemple dans désir-eux, où la succession des deux lettres $r$ (du signe désir) et $e$ (du signe -eux) a exactement le même statut - linéaire - que la succession de $d$ et de é dans le signe désir. En sorte que la caractère linéaire du signifiant entraîne de façon à la fois nécessaire et subreptice le caractère linéaire de l'enchaînement des signes, quelle que soit la dimension de ces signes: "sous-unités" telles que désir et - eux, mots, syntagmes, etc.

5. /.../ mais, on le sait, pas toujours: il arrive parfois que l'anagramme prenne une forme phrastique. Voir Arrivé 1986 a.

6. Mention à peu près unique, dans la recherche anagrammatique, du nom de la linguistique.

7. On constate qu'ici c'est bien le problème du caractère linéaire du signifiant au sens strict (« les éléments qui forment un mot»), et non celui de l'enchaînement linéaire des signes dans le syntagme.

8. «Phrase inachevée dans le manuscrit » (note de Starobinski).

9. On remarquera avec intérêt qu'Hagene n'est pas le seul personnage illustre à se trouver anagrammatisé dans ce vers : on y décryptera aussi celui ... d'Hagège.

10. La formule de Marinetti et Melo se contente de signaler l'exclusion: "Le restanti pagine contengono operazioni anagrammatiche relative a versi del Nibelungenlied ». Tout arbitraire que parait cette exclusion, elle me semble cependant répondre à une analyse, malheureusement laissée implicite, analogue à la mienne : la pratique anagrammatique n'est pas considérée par Saussure comme fondamentale dans le Nibelungenlied. 
11. Je précise toutefois que, comme on a vu, cette disjonction s'observe non dans l'objet même, mais dans le traitement auquel il est soumis. 